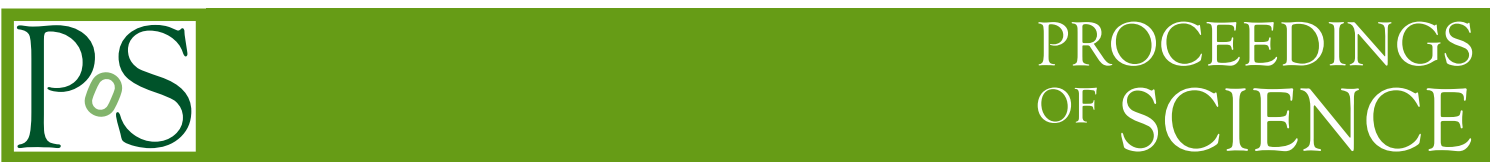

\title{
Status of three-neutrino oscillations
}

\section{Michele Maltoni*}

Instituto de Física Teórica UAM/CSIC,

Calle de Nicolás Cabrera 13-15, Ciudad Universitaria de Cantoblanco, E-28049 Madrid, Spain

E-mail: michele.maltoni@csic.es

We present an up-to-date global analysis of solar, atmospheric, reactor and accelerator neutrino data in the framework of three-neutrino oscillations. We discuss in detail the statistical significance of the observed "hint" of non-zero $\theta_{13}$ in the solar sector at the light of the latest experimental advances, such as the Borexino spectral data, the lower value of Gallium rate recently measured in SAGE, and the low energy threshold analysis of the combined SNO phase I and phase II. We also study the robustness of the results under changes of the inputs such as the choice of solar model fluxes. In the atmospheric sector we focus on the latest results for $v_{e}$ appearance from T2K and MINOS and on the recent Super-Kamiokande results from the combined phases I, II and III, and we discuss their impact on the determination of $\theta_{13}$. Finally, we combine all the data into a global analysis and determine the presently allowed ranges of masses and mixing.

The 2011 Europhysics Conference on High Energy Physics, EPS-HEP 2011,

July 21-27, 2011

Grenoble, Rhône-Alpes, France

\footnotetext{
${ }^{*}$ Speaker.
} 


\section{Introduction}

It is now an established fact that neutrinos are massive and leptonic flavors are not symmetries of Nature. In the last decade this picture has become fully proved thanks to the upcoming of a set of precise experiments. In particular, the results obtained with solar and atmospheric neutrinos have been confirmed in experiments using terrestrial beams: neutrinos produced in nuclear reactors and accelerators facilities have been detected at distances of the order of hundreds of kilometers.

The minimum joint description of all the neutrino data requires mixing among all the three known neutrinos $\left(v_{e}, v_{\mu}, v_{\tau}\right)$, which can be expressed as quantum superpositions of three massive states $v_{i}(i=1,2,3)$ with masses $m_{i}$. This implies the presence of a leptonic mixing matrix in the weak charged current interactions which can be parametrized as:

$$
U=\left(\begin{array}{ccc}
1 & 0 & 0 \\
0 & c_{23} & s_{23} \\
0 & -s_{23} & c_{23}
\end{array}\right) \cdot\left(\begin{array}{ccc}
c_{13} & 0 & s_{13} e^{-i \delta_{\mathrm{CP}}} \\
0 & 1 & 0 \\
-s_{13} e^{i \delta_{\mathrm{CP}}} & 0 & c_{13}
\end{array}\right) \cdot\left(\begin{array}{ccc}
c_{21} & s_{12} & 0 \\
-s_{12} & c_{12} & 0 \\
0 & 0 & 1
\end{array}\right) \cdot\left(\begin{array}{ccc}
e^{i \eta_{1}} & 0 & 0 \\
0 & e^{i \eta_{2}} & 0 \\
0 & 0 & 1
\end{array}\right)
$$

where $c_{i j} \equiv \cos \theta_{i j}$ and $s_{i j} \equiv \sin \theta_{i j}$. In addition to the Dirac-type phase $\delta_{\mathrm{CP}}$, analogous to that of the quark sector, there are two physical phases $\eta_{i}$ associated to the Majorana character of neutrinos and which are not relevant for neutrino oscillations.

Given the observed hierarchy between the solar and atmospheric mass-squared splittings there are two possible non-equivalent orderings for the mass eigenvalues, which can be associated to the two possible choices of the sign of $\Delta m_{31}^{2}$ and are usually denoted as normal scheme $\left(\Delta m_{31}^{2}>0\right)$ and inverted scheme $\left(\Delta m_{31}^{2}<0\right)$. In this convention the angles $\theta_{i j}$ can be taken without loss of generality to lie in the first quadrant, $\theta_{i j} \in[0, \pi / 2]$, and the phases $\delta_{\mathrm{CP}}, \eta_{i} \in[0,2 \pi]$.

Thanks to the synergy amongst a variety of experiments involving solar and atmospheric neutrinos, as well as man-made neutrinos at nuclear power plants and accelerators, we have now a relatively detailed picture of the parameters describing three-flavor neutrino oscillations.

\section{Leading $\Delta m_{21}^{2}$ oscillations: solar and KamLAND data}

In the analysis of solar neutrino experiments we include the total rates from the radiochemical experiments Chlorine [1], Gallex/GNO [2] and SAGE [3]. For real-time experiments we include the 44 data points of the electron scattering (ES) Super-Kamiokande phase I (SK-I) energy-zenith spectrum [4] and the data from the three phases of SNO [5, 6, 7], including the results on the low energy threshold analysis of the combined SNO phase I and phase II [8] (which we label SNO-LETA). We also include the main set of the 192 days of Borexino data [9] as well as their high-energy spectrum from 246 live days [10].

In Fig. 1(a) we show the present determination of the leading parameters $\Delta m_{21}^{2}$ and $\theta_{12}$ from the analysis of the KamLAND data [11] as well as the updated oscillation analysis of the solar neutrino data described above in the context of the GS98 solar model. For comparison we also show the results obtained prior to the inclusion of the energy spectrum of Borexino $[9,10]$ and 

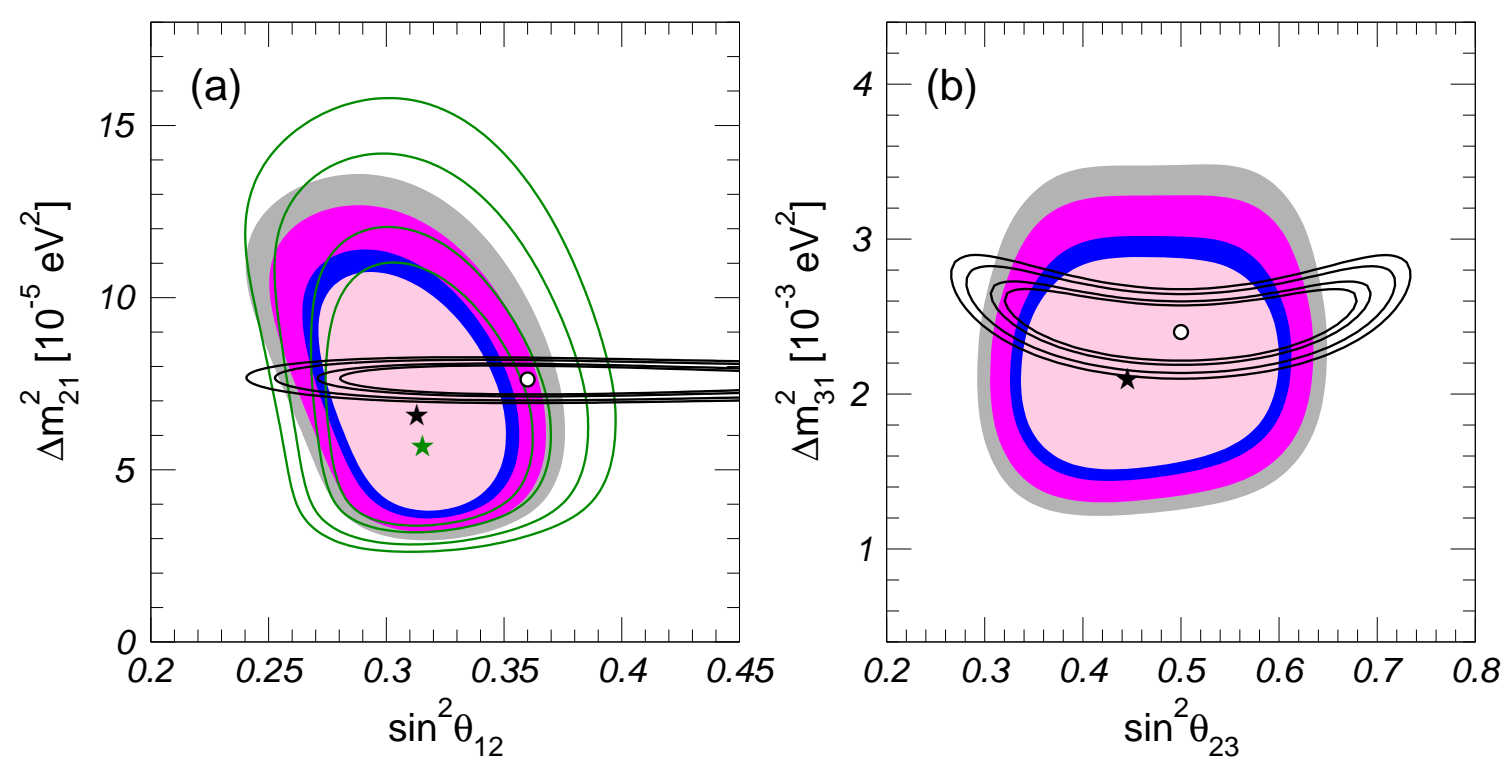

Figure 1: Allowed parameter regions (at 90\%, 95\%, 99\% and 99.73\% CL for 2 d.o.f.) for $\theta_{13}=0$. Left (a): analysis of KamLAND (black lines) and solar data assuming the GS98 solar model, with (full regions) and without (green lines) the inclusion of Borexino data and the low energy threshold analysis of the combined SNO phase I and phase II. Right (b): analysis of atmospheric data (full regions) and LBL data (black lines) for $\Delta m_{21}^{2}=7.6 \times 10^{-5} \mathrm{eV}^{2}$.

the SNO-LETA results [8] for the same solar model. As seen in this figure, the inclusion of these results lead to an improvement on the determination of both $\theta_{12}$ and $\Delta m_{21}^{2}$ and for this last one the best-fit value slightly increases. The most quantitatively relevant new information arises from the inclusion of the SNO-LETA results. The inclusion of Borexino tends to shift the region towards slightly lower values of $\theta_{12}$ angle. Conversely, if the analysis is done in the context of the AGSS09 model [12] the region is shifted towards slightly larger $\theta_{12}$.

\section{Leading $\Delta m_{31}^{2}$ oscillations: atmospheric and accelerator data}

In the analysis of atmospheric data we include the results of the first three runs of SuperKamiokande, denoted as SK(I+II+III) [13]. For details on our simulation of the data samples and the statistical analysis see the Appendix of Ref. [14]. Concerning LBL accelerator experiments we combine the results on $v_{\mu}$ disappearance from K2K [15] with those obtained by MINOS in the $v_{\mu} \rightarrow v_{\mu}$ channel $\left(7.2 \times 10^{20}\right.$ p.o.t. $)[16], \bar{v}_{\mu} \rightarrow \bar{v}_{\mu}$ channel $\left(2.9 \times 10^{20}\right.$ p.o.t. $)$ [17] and $v_{\mu} \rightarrow$ $v_{e}$ channel $\left(8.2 \times 10^{20}\right.$ p.o.t. $)$ [18]. We also include the recent $v_{\mu} \rightarrow v_{e}$ appearance results from T2K [19].

In order to test the description of the present data in the absence of $\theta_{13}$-induced effects we show in Fig. 1(b) the present determination of the leading parameters $\Delta m_{31}^{2}$ and $\theta_{23}$ for $\theta_{13}=0$ and $\Delta m_{21}^{2}=7.6 \times 10^{-5} \mathrm{eV}^{2}$. For concreteness we plot only normal ordering; the case of inverted ordering gives practically identical results as long as $\theta_{13}=0$. This figure illustrates how the bounds 


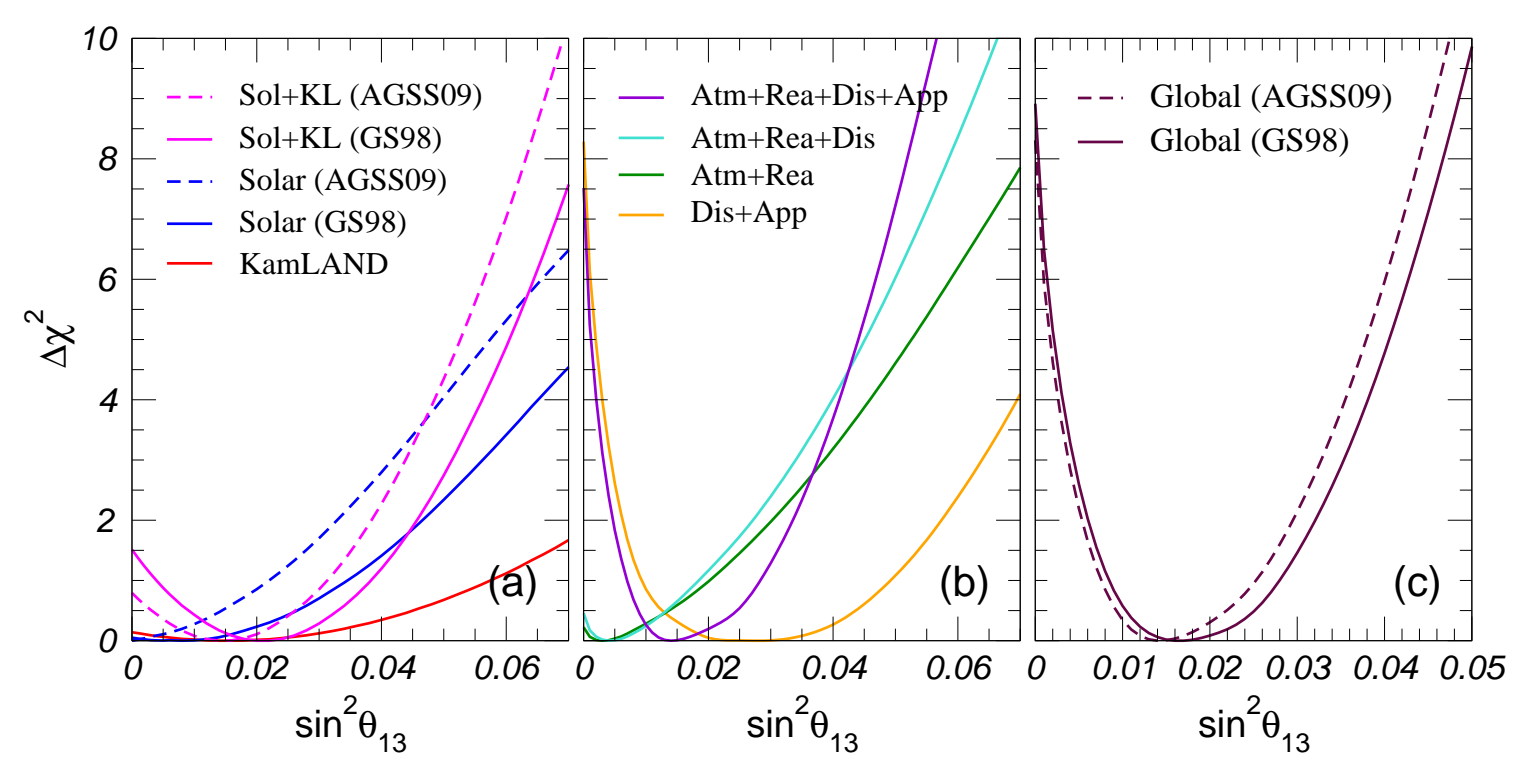

Figure 2: $\Delta \chi^{2}$ dependence on $\sin ^{2} \theta_{13}$ from various data sets as labeled in the figure. AGSS09 and GS98 refer to low and high metallicity solar models, respectively.

on the oscillation parameters $\theta_{23}$ and $\Delta m_{31}^{2}$ emerges from a complementarity of atmospheric and accelerator neutrino data: $\left|\Delta m_{31}^{2}\right|$ is determined by the spectral data from MINOS, whereas the mixing angle $\theta_{23}$ is still dominated by atmospheric data from Super-Kamiokande with a bestfit point close to maximal mixing. Note that there is a small tension between SK(I+II+III) and MINOS, as the first sample prefers a lower value of $\left|\Delta m_{31}^{2}\right|$ than the second one.

\section{Status of $\theta_{13}$ from global data}

The third mixing angle $\theta_{13}$ is of crucial importance for future oscillations experiments. Fig. 2 summarizes the information on $\theta_{13}$ from present data, which emerges from an interplay of different data sets. An important contribution to the bound comes, of course, from the $\mathrm{CHOOZ}$ reactor experiment [20] combined with the determination of $\left|\Delta m_{31}^{2}\right|$ from atmospheric and long-baseline experiments. Another indication of non-zero $\theta_{13}$ arises from the results of the MINOS and T2K $v_{\mu} \rightarrow v_{e}$ appearance data. Recently the T2K collaboration announced the observation of $6 v_{e}$-like events in the Super-Kamiokande detector associated to the Tokai $v_{\mu}$ beam, whereas the expectation in absence of oscillation is just $1.5 \pm 0.3$. This gives a $2.5 \sigma$ indication in favor of non-zero $\theta_{13}$ [19], which adds to the $1.6 \sigma$ hint observed by MINOS [18].

An important piece of information on $\theta_{13}$ comes from solar and KamLAND data. The relevant survival probabilities imply an anti-correlation of $\sin ^{2} \theta_{13}$ and $\sin ^{2} \theta_{12}$ for KamLAND and low energy solar neutrinos, and a positive correlation for the high energy part of the solar spectrum. This complementarity leads to a non-trivial constraint on $\theta_{13}$ and it allows to understand the hint for a non-zero value of $\theta_{13}$, which helps to reconcile the slightly different best fit points for $\theta_{12}$ as well as for $\Delta m_{21}^{2}$ for solar and KamLAND separately. 
We find that the inclusion of the new solar data, and in particular of the SNO-LETA results tends to lower the statistical significance of $\theta_{13} \neq 0$, while the results from $v_{e}$ appearance from MINOS and T2K increase it. Within the context of the solar model with higher metallicities (GS98) we conclude that the significance of $\theta_{13} \neq 0$ from the global analysis has now reached the $3.0 \sigma$ level, mainly driven by the strong $\mathrm{T} 2 \mathrm{~K}$ result. Using the solar neutrino fluxes required to fit the lower metallicity data (AGSS09) slightly lowers the statistical significance $(2.9 \sigma)$ and the best fit value of $\theta_{13}$.

\section{Acknowledgments.}

This work is supported by Spanish MICINN grants FPA-2009-08958, FPA-2009-09017 and consolider-ingenio 2010 grant CSD-2008-0037, by Comunidad Autonoma de Madrid through the HEPHACOS project S2009/ESP-1473, and by the EU grant EURONU.

\section{References}

[1] B. T. Cleveland et al., Astrophys. J. 496 (1998) 505.

[2] F. Kaether et al., Phys. Lett. B 685 (2010) 47 [arXiv:1001.2731].

[3] J. N. Abdurashitov et al. [SAGE collab.], Phys. Rev. C 80 (2009) 015807 [arXiv:0901.2200].

[4] J. Hosaka et al. [Super-Kamkiokande collab.], Phys. Rev. D 73 (2006) 112001 [hep-ex/0508053].

[5] B. Aharmim et al. [SNO collab.], Phys. Rev. C 75 (2007) 045502 [nucl-ex/0610020].

[6] B. Aharmim et al. [SNO collab.], Phys. Rev. C 72 (2005) 055502 [nucl-ex/0502021].

[7] B. Aharmim et al. [SNO collab.], Phys. Rev. Lett. 101 (2008) 111301 [arXiv:0806.0989].

[8] B. Aharmim et al. [SNO collab.], Phys. Rev. C 81 (2010) 055504 [arXiv:0910.2984].

[9] C. Arpesella et al. [Borexino collab.], Phys. Rev. Lett. 101 (2008) 091302 [arXiv:0805.3843].

[10] G. Bellini et al. [Borexino collab.], Phys. Rev. D 82 (2010) 033006 [arXiv:0808.2868].

[11] I. Shimizu [KamLAND collab.], J. Phys. Conf. Ser. 120 (2008) 052022.

[12] A. Serenelli et al., Astrophys. J. 705 (2009) L123 [arXiv:0909.2668].

[13] R. Wendell et al. [Super-Kamiokande collab.], Phys. Rev. D 81 (2010) 092004 [arXiv:1002.3471].

[14] M. C. Gonzalez-Garcia and M. Maltoni, Phys. Rept. 460 (2008) 1 [arXiv:0704.1800].

[15] M. H. Ahn et al. [K2K collab.], Phys. Rev. D 74 (2006) 072003 [hep-ex/0606032].

[16] A. Himmel, talk presented at the Fermilab National Accelerator Laboratory, June 14, 2010.

[17] A. Sousa, talk presented at the Fermilab National Accelerator Laboratory, August 25, 2011.

[18] P. Adamson et al. [MINOS collab.], Phys. Rev. Lett. 107 (2011) 181802 [arXiv:1108.0015].

[19] K. Abe et al. [T2K collab.], Phys. Rev. Lett. 107 (2011) 041801 [arXiv:1106.2822].

[20] M. Apollonio et al. [CHOOZ collab.], Eur. Phys. J. C 27 (2003) 331 [hep-ex/0301017]. 